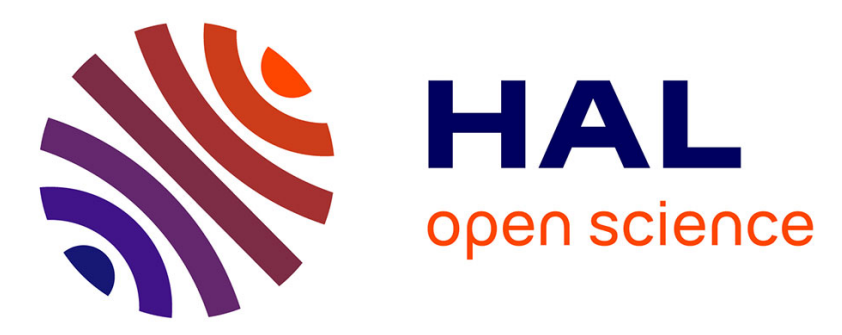

\title{
La visite scolaire: un espace singulier au croisement de deux institutions
}

Cora Cohen-Azria, Ana Dias-Chiaruttini

\section{To cite this version:}

Cora Cohen-Azria, Ana Dias-Chiaruttini. La visite scolaire: un espace singulier au croisement de deux institutions. Cohen-Azria C., Chopin M.-P., Orange D. Questionner l'espace Les méthodes de recherche en didactiques, Presses universitaires du Septentrion, pp.133-148, 2016. hal-01613220

\section{HAL Id: hal-01613220 \\ https://hal.science/hal-01613220}

Submitted on 9 Oct 2017

HAL is a multi-disciplinary open access archive for the deposit and dissemination of scientific research documents, whether they are published or not. The documents may come from teaching and research institutions in France or abroad, or from public or private research centers.
L'archive ouverte pluridisciplinaire HAL, est destinée au dépôt et à la diffusion de documents scientifiques de niveau recherche, publiés ou non, émanant des établissements d'enseignement et de recherche français ou étrangers, des laboratoires publics ou privés. 


\title{
La visite scolaire : un espace singulier au croisement de deux institutions
}

\author{
Cora Cohen-Azria
}

Ana Dias-Chiaruttini

Théodile-CIREL EA 4354, Université de Lille 3

Nous nous proposons dans ce chapitre d'aborder la question des espaces dans le cadre de la visite scolaire au musée. Celle-ci se déroulant pendant le temps scolaire, dans une institution autre que l'école, son analyse soulève ainsi d'un point de vue méthodologique la question des espaces et de leurs découpages. Ces derniers sont au moins de trois ordres :

- l'espace-temps, quand commence la visite ? quand se termine-t-elle?

- l'espace géographique, comment définir ses bornes ? ses frontières?

- l'espace que nous nommons « institutionnel» pour délimiter entre autres la nature des savoirs de référence en jeu lors de la visite (à quelle institution se réfèrent-ils ?) mais aussi les contenus visés, construits, apportés, transformés par la situation et les sujets que nous observons (quels sont les sujets institués, convoqués par cette situation? un élève ? un visiteur? un élève-visiteur (Cohen-Azria, 2002 ; 2011), etc.) ?

La question du découpage est essentielle, elle nous permet de délimiter, de borner la visite scolaire et de déterminer ce que nous choisissons d'analyser comme données pour la caractériser et ce que nous écartons (Cohen-Azria, 2012).

Notre propos s'enracine sur deux recherches ${ }^{1}$ que nous menons ensemble au sein de Théodile-CIREL (Cohen-Azria, Dias-Chiaruttini, 2013 ; Cohen-Azria, Dias-Chiaruttini, 2015) et qui nous permettent de croiser nos approches de didacticiennes de deux disciplines différentes : nous sommes en effet didacticienne des Sciences pour l'une et didacticienne du Français pour l'autre.

Nous nous appuierons principalement sur un questionnaire diffusé auprès d'élèves ${ }^{2}$ et des observations de visites réalisées dans trois espaces muséaux différents de la région du Nord de la France (Musée d'histoire naturelle, LaM - Musée d'art moderne et d'art brut - et Forum départemental des sciences), ainsi que des observations faites en classe.

Notre réflexion s'articule autour de trois points: la question des découpages des situations ; celle des découpages disciplinaires interrogeant ainsi la nature des savoirs; et la question du sujet didactique, en d'autres termes, l'étude des statuts des sujets.

\footnotetext{
${ }^{1}$ La première porte sur Expériences de visites au musée: pratiques et discours des sujets et la seconde sur Visites scolaires, péri-extrascolaires et familiales dans des lieux muséaux de la région Nord-Pas-de-Calais : approches didactiques.

${ }^{2}$ Ce questionnaire a été transmis aux élèves par leur enseignant à la suite de la visite et complété en classe. Il nous était renvoyé par la poste par les enseignants via une enveloppe pré-timbrée qui leur était remise avec les exemplaires du questionnaire pour chaque élève. Il a été diffusé auprès de toutes les classes visitant les espaces muséaux associés à la recherche par les responsables du service pédagogique qui ont été nos partenaires. Nous les remercions de ce soutien et de leur investissement.
} 


\section{Espaces géographiques : découpages des situations}

Avant de présenter certaines questions que nous pose le découpage des situations, précisons ce que nous entendons par situation et plus spécifiquement par situation didactique. Nous considérons en effet la visite scolaire comme une situation didactique (Lahanier-Reuter, 2007). En cela elle est une " coupe » dans la réalité de la vie de la classe qui permet de saisir ce qui émerge de nouveau en termes d'enseignements et d'apprentissages dans cet espace spatial et temporel ainsi découpé. Nous considérons que la visite scolaire au musée fait émerger des contenus spécifiques, situe les sujets didactiques (les place dans une situation) et favorise des sensibilisations ou des enseignements et / ou des apprentissages qui lui sont spécifiques.

Poser la visite scolaire au musée comme une situation didactique induit une approche méthodologique et théorique de notre objet de recherche qui se différencie d'autres travaux. Ainsi en didactique du Français, les chercheurs s'intéressant à l'œuvre d'art, analysent les conditions et les produits de sa réception (Chabanne, 2013), le musée devient un espace de réception qui favorise une expérience essentiellement esthétique et induit une forme de discours sur l'œuvre. L'expérience de la visite du musée et du contexte scolaire de cette visite n'est pas l'enjeu principal de ces travaux, même si la réception de l'œuvre d'art pose indéniablement la question du contexte. De même, en didactique des sciences, à côté des recherches sur l'espace scolaire, les travaux sur les musées se sont développés très tôt et avec évidence. En effet, pour Clément (1998) concernant la biologie, dans la mesure où les médias qui vulgarisent cette discipline sont pensés comme des sources d'informations précieuses pour les enseignants et les élèves, il s'avère nécessaire, d'une part, d'apprendre à ces sujets à en avoir une utilisation critique et, d'autre part, de développer les recherches en didactique pour analyser le fonctionnement de ces médias spécifiques. La place de la didactique des sciences semble ici davantage s'approcher des questionnements de médiatique (Guichard, Martinand 2000), pour autant les recherches s'inscrivent explicitement en didactique des sciences. En effet, celle-ci a investi les espaces muséaux considérant qu'il s'agissait de «lieux de transmission et d'appropriation de connaissances » relatives aux disciplines scientifiques, d'autant que les apprentissages formels, même si premiers dans l'histoire des questionnements didactiques, ne sont pas exclusifs en termes d'espace d'investigations didactiques (Clément, 1998, p. 129). Ces recherches ne se focalisent alors pas sur la construction de savoirs par les visiteurs, elles incluent tous les types d'acteurs (concepteurs, visiteurs, etc.) mais également les processus en jeu dans l'écriture et la lecture muséographique (par exemple : Guichard, 1989, Triquet et Clément, 1990, etc.). Lorsque ces recherches se focalisent sur les visites scolaires, elles développent finalement leurs questionnements et leurs méthodologies comme elles le font dans la classe et ce, de manière implicite.

Si on tente de décrire la visite scolaire au musée comme une situation didactique alors une série de questions se pose à nous :

La visite scolaire commence-t-elle au moment de l'annonce de la sortie ? Ou à la sortie physique de l'école ? Ou à l'entrée au musée ? Ou dans celle de l'exposition ? Le temps du déplacement est-il un temps a-didactique ou didactique ? Peut-il être considéré comme un moment, une situation sans contenu, ou est-il un espace de transition qui agit sur les sujets et par voie de conséquence sur les contenus de la visite scolaire?

Nous considérons que les espaces géographiques ne sont pas suffisants pour rendre compte des contenus des situations de visite scolaire au musée. Nous pensons ainsi que les espaces de transition ne sont pas à exclure de la situation de visite, qu'ils agissent sur elles, y participent et la contraignent. Cependant ils constituent des espaces souvent ignorés dans les travaux portant sur la visite au musée. 
Ce choix méthodologique nous permet :

- de penser la situation de visite scolaire dans un « continuum didactique ». La visite s'inscrit alors dans l'histoire de classe, l'histoire scolaire et quel que soit le traitement qui est fait de ce moment, il appartient à une temporalité avec un avant et un après la visite sans $a$ priori contraindre le découpage analytique ;

- de repenser les liens entre les institutions scolaire et muséale au sein de cette situation didactique qui dans de nombreux travaux se trouvent en opposition (Jacobi, Coppey, 1995) visant des contenus (plaisir vs ennui) et la formation/conception d'un sujet différent (visiteur $v s$ élève) pensés en tension.

Dans le cadre de la recherche Expériences de visite au musée : pratiques et discours des sujets, nous avons ainsi diffusé des questionnaires auprès des élèves leur permettant de s'exprimer entre autres sur les liens qu'ils reconstruisent entre les apprentissages scolaires et la visite muséale ou encore sur les spécificités de cette visite par rapport à d'autres expériences de visites muséales. Nous articulons l'analyse de ces discours aux observations que nous avons faites de ces visites. Celles-ci s'appuient en particulier sur des données vidéo que nous avons construites.

Nous avons fait le choix de filmer en caméra mobile, des plans larges avec des zooms sur des moments d'interactions spécifiques entre le guide et certains élèves. Ces choix en euxmêmes opèrent des découpages dans ce que nos observations permettent d'analyser de la situation. Les documents vidéo construits ne donnent pas à voir la scénographie ni les choix muséographiques mais s'attardent sur le découpage réalisé par la visite scolaire dans cet espace écrit. Pour autant, l'analyse de la lecture réalisée par le groupe scolaire ne peut se faire sans la prise en compte de la scénographie de l'exposition. Par exemple, au LaM nous ne filmons aucune salle d'exposition ni les œuvres qu'elles soient objet d'étude ou pas. Elles peuvent apparaitre dans le champ de la caméra, mais elles ne sont pas constituées comme un élément premier de nos observations. Nous avons fait le choix de nous centrer sur les sujets, l'un de nos objets d'analyse étant les interactions observables (partagées au sein du groupeclasse). Nous filmons cependant les déplacements à travers le musée, d'une salle à l'autre, et observons les postures corporelles des élèves, des enseignants, du guide. Ce qui nous amène à une double retranscription des données ainsi recueillies: les échanges verbaux et les mouvements des corps et du groupe classe. Ceci nous permet d'avoir une lecture plurielle de la situation de visite. C'est ainsi qu'une écoute à l'aveugle de la vidéo au $\mathrm{LaM}^{3}$ nous a permis de constater le peu de différence dans les interactions en classe et au musée. Les échanges sont insuffisants pour déterminer que l'une des deux situations se déroule au musée. La prise en compte de l'espace, du corps des élèves, assis par terre, allongés, se redressant, levant la main pour prendre la parole, se bousculant, riant dans les déplacements sont autant d'indices de la situation qui nous permettent de caractériser la visite scolaire comme n'étant pas une situation scolaire en soi (élèves allongés devant l'œuvre d'art), et comme n'étant pas une situation de visite que nous qualifierons ici d'extrascolaire ou familiale (élève levant la main pour répondre aux questions du guide). Cependant nous traitons inégalement cette double lecture (des interactions et des mouvements corporels) qui se situe à des degrés différents. En effet, notre analyse porte d'abord sur les interactions et se réfère aux mouvements des corps dans des cas spécifiques de notre réflexion (Veron, Levasseur, 1983). Nous ne les prenons en compte que lorsqu'ils permettent de caractériser la visite scolaire. Ainsi, si les échanges verbaux mis en commun sont tous retranscrits (dans la mesure du possible), tous les mouvements corporels, toutes les postures ne le sont pas.

\footnotetext{
${ }^{3}$ Musée d'art moderne et d'art brut et le forum des sciences, tous deux à Villeneuve d'Ascq
} 
Dans le cadre de la recherche sur Les visites scolaires, péri-extrascolaires et familiales dans des lieux muséaux de la région Nord-Pas-de-Calais nous avons fait d'autres choix méthodologiques pour nos observations. Cette recherche a pour finalité de confronter différents types de visites auxquelles participent des élèves ou des enfants pour voir s'ils se construisent comme visiteurs, et voir aussi ce qui est spécifique à la visite scolaire. Comment filmer ces différents contextes de visite aux musées afin de permettre une confrontation? Comment filmer une visite familiale? Faut-il filmer tous les membres d'une famille? Comment prendre en compte que le groupe de visiteurs que constitue une famille se déplace autrement qu'une classe, adopte un autre rythme, et réalise des parcours différents ? Comment prendre en compte que tantôt l'enfant peut accompagner l'adulte ou tantôt c'est l'adulte qui accompagne l'enfant? Ou encore que dans le cadre des visites réalisées pendant le temps des centres aérés, les parcours sont différents, les déambulations dans l'espace aussi. Nous avons fait le choix de filmer la visite singulière d'un sujet enfant ou élève pour chaque type de visite que nous réalisons à l'aide d'une caméra GoPro. Nous faisons dans ce cas le deuil de l'observation des sujets visitant un musée pour constituer comme objet d'observation et de recherche la visite réalisée par un sujet interagissant avec d'autres. Cependant que reconstituons-nous de la visite et du sujet qui nous permet d'observer son parcours de visite ? Ce choix méthodologique nous amène à des bougés considérables dans la reconstruction que nous faisons de la visite muséale dans divers contextes sociaux (scolaires, extrascolaires, familiaux). Les retranscriptions, par exemple, sont découpées dans le cadre de la recherche sur Expériences de visite au musée: pratiques et discours des sujets en fonction des déplacements qui rythment à chaque fois la découverte d'une œuvre au musée du LaM. Ce découpage qui nous permettait de faire un rapide synopsis des visites scolaires dans ce musée ne peut plus être retenu dans le cadre de notre seconde recherche et nous sommes confrontées à d'autres choix de repères pour décrire la visite familiale, scolaire et extrascolaire telle que nous l'observons sous ce nouveau point de vue.

Ces réflexions posent par ailleurs la question de l'espace de la visite scolaire par rapport à l'espace de la visite au musée de publics autres que scolaires. D'une part l'espace de visite scolaire est différent de celui des visites conçues pour les autres publics, ce qui suppose une prise en compte spécifique du public scolaire et des médiations scientifiques ou culturelles faites dans ce cadre. Nos observations permettent de décrire ces variations des espaces et des parcours de visites des musées selon les contextes de visites.

D'autre part les travaux ont montré qu'il existe des espaces réservés aux publics scolaires (Cohen et Girault, 1999). Ainsi par exemple, dans l'observation réalisée au LaM ou au musée d'histoire naturelle, après la visite de l'exposition, il est proposé aux élèves un atelier dans une salle spécifique. Prolonge-t-il la visite de l'exposition ? Prépare-t-il le retour en classe ? S'adresse-t-il au groupe classe ou à chacun des visiteurs qui le constitue? Comment prendre en compte cet autre lieu et cet autre moment de la visite au musée ? Dans la continuité avec l'exposition, dans la rupture, comme un espace transitoire ?

Le choix que nous avons fait d'analyser la visite scolaire au musée comme une situation didactique nous permet de questionner explicitement les choix des découpages que nous faisons et leur incidence sur les analyses construites. Ce choix nous permet notamment de ne pas restreindre nos observations à la visite de l'exposition et d'intégrer des espaces autres que celui de l'exposition. Il nous permet aussi de confronter des contextes de visites : scolaires, extrascolaires, familiales et prendre en compte leurs effets sur l'espace de la visite et notamment sur le parcours et les lieux du musée investis par chaque visite (DiasChiaruttini, 2012). 


\section{Espaces disciplinaires : nature des savoirs}

Si les découpages entre disciplines structurent l'organisation scolaire, les Musées peuvent chercher à s'en distinguer. Parfois pour se mettre à distance de la culture scolaire, parfois aussi parce que ce découpage ne convient pas aux contenus qu'ils construisent et présentent. Quoi qu'il en soit, les visites scolaires peuvent exploiter l'espace muséal en réinjectant des découpages disciplinaires à différents niveaux : dans les interprétations des expositions; dans les cheminements physiques pensés à travers les objets afin de construire un discours particulier; dans les ateliers ou animations jouxtant les expositions. Ces découpages se retrouvent mobilisés par le musée lui-même, notamment dans l'écriture des programmes en direction des scolaires ou encore dans les formations continues en direction des enseignants au sein des musées.

Notre réflexion nous amène à poser la question des savoirs de référence des deux institutions (scolaire et muséale) dans la conception des contenus de la visite scolaire. Sont-ils des contenus muséaux, ou scientifiques muséographiés ou artistiques muséographiés? Contenus scolaires, ou scientifiques scolarisés, ou artistiques scolarisés ? Ou les deux selon les lieux et les moments ? Ou encore des contenus scientifiques, artistiques muséographiés puis scolarisés?

Pour avancer dans ce questionnement, nous avons confronté des situations de lecture d'œuvres d'art effectuées au musée ou en classe pour caractériser ce qu'elles ont de spécifiques outre le mode de présence de l'œuvre d'art. L'analyse peut alors porter sur les interactions médiateur - élèves, enseignant - élèves, et élève - élève et les contenus mobilisés par ces échanges ${ }^{4}$. L'analyse des interactions montre des formes d'échanges très similaires. Le schéma de communication apparaît peu différenciable dans les deux situations filmées : en classe comme au musée, les élèves lèvent la main pour prendre la parole ; en classe comme au musée, les élèves répondent aux questions de l'enseignant ou du guide; pratiquement aucune interaction entre élèves face aux œuvres n'est observable dans les deux espaces (DiasChiaruttini, 2015). Les discours sur l'œuvre se structurent au musée, comme en classe, autour de trois formes d'activités langagières : descriptions, émission d'hypothèses et interprétations. Est-ce la forme de la communication scolaire qui se déplace au musée ? Est-ce une forme du discours sur l'œuvre d'art qui se déplace d'une institution à l'autre, ou qui serait partagée entre ou au-delà des institutions? Chabanne et Dufays $(2011$, p. 11 - 12) considèrent qu'en « situation scolaire, la parole s'insère dans une interaction didactique, où interviennent de manière décisive non seulement la parole du maître, mais aussi de nombreux discours de référence, qui servent de modèles implicites à ce qu'on attend des élèves ». L'analyse du discours construit face à une œuvre d'art au musée, ou face à sa reproduction en classe, montre des références communes qui relèvent de l'Histoire des arts, en tant que discipline de recherche: connaissances sur les peintres (leur vie, leur œuvre), sur les mouvements artistiques; sur les techniques de composition des œuvres lues; sur les effets de réception attendus. En classe, la lecture part de la composition d'ensemble vers l'explicitation de détails observés, au musée, nous observons le mouvement inverse, centration sur des détails pour reconstruire la composition globale de l'œuvre. Néanmoins ces choix peuvent davantage être liés aux œuvres elles-mêmes plutôt qu'aux institutions : la lecture d'une œuvre d'art moderne nécessitant certaines formes de décodages de ce qui est représenté et de leur signification.

Les mouvements sémiotiques des corps des élèves sont par contre révélateurs de différences entre les deux espaces institutionnels : face à l'œuvre d'art au musée, les élèves

\footnotetext{
${ }^{4}$ Nous prenons appui sur un travail en partenariat avec le musée du LaM et des observations de lecture d'œuvres d'art réalisées en classe de CM2.
} 
sont - à la demande du guide - assis par terre et les corps s'allongent, se redressent, s'étirent. Des rires étouffés, des sourires, des regards sont différents de ce qui peut être observé en classe.

Le discours du guide se spécifie aussi dans le parcours de visite qui est proposé construisant une culture du musée : «on va commencer par regarder ce tableau qui est fait avec de la peinture, par qui a-t-il été peint ? Est-ce que vous le savez ? Comment on fait pour le savoir dans un musée ? » Il construit ainsi le rôle du cartel mais aussi un comportement de visiteur de musée attendu. Les déplacements permettent au guide de familiariser les élèves avec un espace particulier et d'observer les choix architecturaux qui ont été faits dans le cadre de l'agrandissement du musée.

Notre analyse a également concerné les interactions au musée d'histoire naturelle. Avant d'aborder la thématique en jeu, ici les fossiles, l'introduction est basée sur le musée à partir d'un questionnement unique posé par le guide, en l'occurrence : que peut-on trouver au musée ? Ce préalable permet de dire quelque chose du lieu, sans évoquer la façon de s'y conduire ou de lire les objets exposés. L'introduction se poursuit sur la thématique des fossiles. Ainsi, il se passera une dizaine de minutes avant que les élèves ne soient invités à observer les vitrines et les objets exposés. Dans cette première partie, comme pour l'extrait précédent, le contenu des retranscriptions ne donne pas à voir la spécificité du lieu. Ainsi, ce questionnement sur ce que les élèves savent des fossiles, de l'âge de la terre, du travail des paléontologues pourrait tout à fait relever d'un espace scolaire introductif à une « leçon », à la manière de ce qui est souvent mis en avant dans le cadre des pédagogies relatives aux sciences. En revanche, dès que les discours s'appuient sur les objets exposés, les contenus se structurent différemment. En effet, l'observation devient le support des discours. On pourrait alors penser qu'il suffirait d'observer en classe des objets similaires ou des reproductions de ces objets pour produire les mêmes types de discours. Mais tel n'est pas le cas. En effet, la muséographie et l'espace scénographié vont alors prendre place dans la structuration des interactions. C'est le cas, par exemple, lorsque les élèves sont invités à observer le fossile de lepidodendron (« arbre avec des écailles » explique le guide en traduisant du grec). En effet, après avoir identifié des «écailles fossilisées", ils lèveront les yeux pour voir la reconstitution d'une partie de l'arbre étudié, ainsi que son contexte de présentation (faune). Les interactions vont alterner systématiquement entre un contenu construit à partir des observations muséographiques, et un autre construit à partir des données apportées par le guide (âge de certains fossiles), avec des incises signées par les élèves (« vous n'avez pas peur qu'on vous les vole? », « une fois j'ai trouvé un fossile d'étoile de mer »).

La plupart des déplacements sont ici introduits par un questionnement posé par le guide : "Alors on va se lever et on va aller voir une autre vitrine qui va nous permettre de comprendre comment se sont formés les fossiles », "A quoi servent les fossiles ? Et bien on va se lever et voir au milieu ». Malgré ces introductions, à chaque déplacement vers une présentation, celle-ci n'est pas systématiquement exploitée. Par exemple, devant la vitrine expliquant la fossilisation, le guide orchestrera les interactions (qui s'avèrent comparables à celles des cours dialogués) durant plus de 11 minutes sans demander l'observation ou l'interprétation de la vitrine. Effectivement, celle-ci ne se prête pas à l'observation par un groupe entier en même temps. Ainsi, il fait le choix de mettre en mots son contenu à la manière de ce qui a été décrit, par ailleurs, dans le cadre de la transposition didactique interne à la classe (depuis un contenu de savoir déterminé, ici dans la vitrine). Le déplacement jusqu'à ce lieu signale la place et le statut qui peuvent être attribués à cette présentation muséographique sans aller au bout de cet enseignement par l'exploitation pourtant attendue par les autres visiteurs. Ici le discours se construit par le questionnement du guide, la sélection qu'il opère dans les réponses, et par les synthèses (prévues en amont) qu'il fait. C'est bien ici 
son exposé aux élèves qui prend le relais de ce qui est exposé aux visiteurs (Lefebvre B., Lefebvre H., 1991).

Mais ce n'est pas toujours le cas, l'exploitation de la vitrine suivante, au contraire, s'appuie sur une observation réalisée par tous les élèves au fur et à mesure. Cela s'explique par le fait que le guide demande dans un premier temps aux élèves de décrire l'objet observé (et non pas dans un premier temps sa mise en scène). Pour faciliter la suite de l'échange, le guide utilise une reproduction plus grande, réalisée sur papier, afin de présenter aux yeux de tous les élèves en même temps l'objet en question concerné.

Ainsi, la conclusion construite à propos de l'observation réalisée au LaM, prend également sens ici. Selon les découpages que nous opérons dans la situation didactique, nous pouvons étudier des interactions spécifiques au fait qu'il s'agisse d'un groupe classe, mais nous pouvons aussi analyser des interactions spécifiques à l'espace muséographique.

Ces choix et analyses nous permettent de penser les contenus en jeu lors de la visite scolaire comme des contenus spécifiques issus de la transformation des savoirs scolaires et muséaux ou comme des contenus nouveaux engendrés par la situation et les acteurs qui la construisent (Cohen-Azria, Dias-Chiaruttini, 2015).

\section{Espaces institutionnels : les statuts des sujets}

La question des sujets est aussi dépendante des découpages que méthodologiquement nous décidons d'opérer. D'un point de vue méthodologique se pose la question de savoir ce que le didacticien observe et étudie : des élèves ? des élèves "disciplinaires »? des élèvesvisiteurs ? des enseignants? des enseignants-visiteurs ? des adultes ? des visiteurs ? etc. Comment reconstruisons-nous ces sujets? Si nous le faisons en référence aux espaces institutionnels, pourquoi en privilégier un aux dépens de l'autre? Si nous opérons une distinction à partir des catégories construites par les sujets adultes en jeu, celle-ci ne peut se construire que dans une tension entre ces deux pôles institutionnels. Le choix que nous avons pour l'instant mis en place et éprouvé est celui de considérer les sujets didactiques dans un espace intermédiaire en construisant le statut d'élève-visiteur (Cohen, 2002). Mais cette construction ne s'appuie pas sur une définition a priori. En effet, il s'agit pour nous de décrire ce statut a posteriori, à partir des données construites. Ainsi, dans le cadre des visites scolaires dans les musées, nous mobilisons cette dénomination afin d'inscrire dans notre discours le questionnement renvoyant le statut des sujets à ces deux espaces. Ainsi, la ou les définition(s) de ce statut intermédiaire résulteront de l'analyse de nos données (observations et questionnaires). Pour autant, considérant les questionnaires adressés aux élèves ayant visité un musée pendant le temps scolaire (cf. annexe), différentes questions d'ordre méthodologique s'imposent à nous.

Tout d'abord, lorsque nous construisons cet outil, comment instituons-nous le sujet? Cette question nous amène à interroger le lieu de la passation (la classe), les relais que nous mobilisons (les enseignants), le texte d'accompagnement et la signature de notre questionnaire (les chercheurs), le contexte de réponse (suite à une visite scolaire au musée), les contextes mobilisés par les questions (les visites scolaires, les visites familiales, les visites avec le centre aéré, etc.). C'est aux élèves que nous nous adressons en premier dans la mesure où c'est en tant qu'élève qu'ils ont réalisé une visite muséale. De plus, ils complètent notre questionnaire à leur retour en classe. Cependant, dans celui-ci, nous leur demandons d'évoquer une expérience de visite scolaire et de la comparer à d'autres expériences de visites menées au musée dans d'autres contextes (familial et extrascolaire). En effet, nous avons choisi de construire cet outil sur un système de redondance : la même question étant déclinée sur plusieurs contextes de visite. Ce cadre stable nous permet d'analyser les productions 
d'élèves dans une comparaison : ce qu'ils disent selon les contextes de visites, mais également la façon qu'ils ont de le dire. Nous cherchons ainsi à convoquer d'autres dimensions que celle du sujet élève à travers ses expériences (Chamberlant, 1991). Les répondants doivent, par exemple, associer des formules aux différents types de visites : lors des visites familiales / scolaires /extrascolaires, «j'ai du plaisir», « je comprends», «je m'ennuie», « je suis fatigué », «ça m'intéresse », «j'apprends » ou encore « on m'explique ». Ces catégories de réponses sont proposées, et nous pouvons, dès lors, étudier si ce sont les mêmes formules qui sont choisies pour qualifier la place des différents sujets selon le type de visite. Mais cette exploration se poursuit également dans l'analyse des réponses aux nombreuses questions ouvertes.

Nous pouvons alors nous demander si c'est toujours le même sujet qui répond au fil du questionnaire ? Peut-on considérer une continuité dans le discours des sujets ou choisit-on de découper les réponses en fonction des contextes de visite mobilisés ? Le premier choix pourrait nous amener à penser des discours d'élèves sur des visites au musée réalisées dans différents contextes. La cohérence du corpus serait alors pensée sur une unité correspondant à chaque questionnaire. La seconde conception considèrerait plutôt des mobilisations de statuts multiples et permettrait de construire une cohérence en fonction des contextes. Ainsi le corpus serait construit à partir de plusieurs sous-corpus : les réponses sur la visite scolaire, celles sur la visite familiale, etc.

L'analyse des contenus des réponses peut alors se poser en terme de lexique spécifique. Existe-t-il pour le chercheur un vocabulaire d'élève et un vocabulaire de visiteur? Ces comparaisons permettent de voir, en effet, si le vocabulaire mobilisé et les idées développées par les répondants le sont de façon contrastée selon le contexte de visite et donc selon la part du sujet dont il est question. Ce questionnement rejoint celui que nous avons concernant nos observations: en quoi certaines postures, certaines activités, ou encore certains comportements peuvent-ils faire référence à l'espace scolaire ou à l'espace muséal ? Dans le cadre des observations, c'est le discours porté par les sujets adultes (enseignants ou médiateurs) qui nous a permis d'avancer dans ce questionnement sans pour autant le clore. Comme nous l'avons vu supra, le guide du LaM s'adresse à une dimension du sujet élève quand il tente de maîtriser les corps et tient certains propos, tels que : « Ici, si l'on veut parler, si l'on veut dire des choses, on lève le doigt. Je veux que tout le monde soit en tailleur, les fesses par terre. »; «J'attends le silence »; « Le doigt! Je n'entends que ceux qui lèvent leur doigt »; «Relevez-vous, on n'est pas à la plage ici ». Au Musée d'histoire naturelle, il est intéressant de voir qu'aucune demande explicite n'est faite aux élèves quant à l'adoption des codes de communication scolaire et pourtant ce sont eux qui s'installent immédiatement. Les élèves vont demander la parole en levant le doigt et attendent (le plus souvent) que le guide les invite à parler. Pour revenir au LaM, en parallèle de cette convocation plus ou moins implicite de l'élève, le guide tente aussi de construire chez eux des comportements de visiteur quand il évoque, par exemple, le rôle du cartel au musée ou encore lorsqu'il sensibilise les élèves à observer l'architecture du Musée :

Le guide : Elles ont plutôt quelle forme, les pièces?

Un élève : Des pavés

Le guide : Des pavés. Bravo. Des cubes, des carrés. Des formes géométriques avec des angles qui sont droits. Quand vous êtes arrivés vous avez vu le musée de dehors. Le musée si on le construisait en petit, on pourrait le construire avec quel jouet?

Un élève : Des cubes 
Le guide : Des cubes, des Lego, des petits cubes. Cette partie du musée... On va aller dans une autre partie et vous allez voir que c'est assez différent. Une dernière question : dans le musée, on a besoin de place pour mettre les cadres, elles sont où les fenêtres ?

Des élèves : En haut

Le guide : Voilà. Elles sont sur le toit. On va avancer, vous allez regarder.

Ainsi l'espace du Musée devient un objet de sensibilisation et de médiation de cette visite scolaire et permet aux élèves de voir le Musée, de s'y déplacer en prenant en compte la spécificité de ce lieu. Le guide au LaM place parfois les élèves dans une situation de spectateur face à une œuvre. Il éduque tant les corps que le regard et nourrit leur imagination pour les rendre sensibles aux œuvres et à leur réception (par exemple une œuvre de Augustin Lesage) :

Le guide : Peut-être, mais en tout cas, il n'a pas appris à faire de la peinture. Il fait de la peinture car il a entendu une voix quand il était dans la mine. Ce n'était pas la voix d'un homme ou d'une femme, c'était la voix de?

Un élève : De dieu.

Le guide : D'un esprit. L'esprit venait le voir, le soir, parce qu'il peignait ou travaillait le soir, et l'aidait à peindre. Au début, ses copains se moquaient de lui et lui disaient qu'il n'était pas peintre, que ce n'était pas son métier, que c'était une lubie, qu'il était mineur. Mais quand ils ont vu les tableaux, ils ont été drôlement impressionnés et n'ont pas cru que c'était lui qui les peignait. Lui, il a dit que ce n'était pas vraiment lui, mais qu'il était guidé par un esprit.

S'il rend les œuvres d'Art Brut accessibles aux élèves en s'adressant à eux dans des termes choisis, prenant souvent des images de leur (présupposé) quotidien, il n'en transmet pas moins des connaissances sur l'œuvre, sur l'artiste, sur les mouvements artistiques, et crée des repères qui peuvent permettre aux élèves de regarder autour d'eux.

En revanche, la partie de la sortie scolaire réalisée dans la salle d'atelier, que ce soit au LaM ou au musée d'histoire naturelle, se construit sur d'autres types d'activités et de savoirfaire et peut-être en considérant un autre sujet. L'analyse de ces extraits permet d'étudier, entre autres, la place et l'activité des différents adultes et des élèves comparativement au moment vécu dans l'exposition. Lorsque les ateliers précèdent les visites, ils ont souvent été décrits dans les analyses didactiques comme des introductions à la visite permettant aux élèves de construire des clefs de lecture mobilisables lors de leur activité de visiteur. Ici la démarche est inverse puisqu'elle prolonge la visite de l'exposition au sein de l'espace muséal (soit par la fabrication de moulage de fossiles au Musée d'histoire naturelle, soit par la composition d'une œuvre préfigurant la maison idéale au LaM). Dans le premier scénario, l'atelier tente de déplacer l'élève en travaillant sur son regard de visiteur, dans le second, s'agit-il de le préparer à son retour en classe ou son retour à la maison ? Les sujets didactiques sont, dans les deux situations observées, invités à réaliser un « objet ». Il aurait été intéressant de suivre leur déplacement et les discours s'y rapportant au-delà des murs du musée. Notre méthodologie n'a pas intégré cette sortie de l'espace muséal en dehors des déclarations consignées dans le questionnaire. Ainsi, à ce jour, nous continuons d'avancer sur la problématique du statut des sujets en nous appuyant sur l'analyse de discours contextualisés ainsi que sur celle des postures et ce, dans une approche comparative.

Dans le cadre de notre recherche sur la visite scolaire au musée, questionner l'espacetemps, l'espace géographique et l'espace « institutionnel» nous a permis de formuler toute 
une série de questions d'ordre méthodologique éclairant ainsi la spécificité du regard didactique. 
Éléments bibliographiques :

Chamberland E. (1991), «Les thèmes de la contextualisation chez les visiteurs de musée », Canadian Journal of Éducation, ${ }^{\circ} 16-4$, p. 292-312.

Chabanne J.-C. \& Dufays J.-L. (Éd.). (2011) "Parler et écrire sur les œuvres : une approche interdidactique des enseignements artistiques et culturels »-Repères 43 (7-29).

Chabanne J.-C. (2013) "Histoire des arts et jeux de langues : comment parler de l'œuvre d'art», Le français aujourd'hui 182 (55-66).

CLÉMENT P. (1993), «La spécificité de la muséologie des sciences et l'articulation nécessaire des recherches en muséologie et en didactiques des sciences », dans Rémus, La Muséologie des sciences et des Techniques, Actes du colloque du 12 et 13 décembre 1991, Dijon, OCIM, p. 128167

CLÉMENT P. (1998), La biologie et sa didactique, dix ans de recherche, Aster, n²7, p. 57-93.

COHEN C. (2002), Quand l'enfant devient visiteur: une nouvelle approche du partenariat École/Musée, Paris, L'Harmattan.

COHEN C., GiRAult Y. (1999), Quelques repères historiques sur le partenariat école-musée ou quarante ans de prémices tombées dans l'oubli. Aster, $\mathrm{n}^{\circ} 29$, p. 12-18.

COHEN-AZRIA C. (2011), Sorties avec l'école dans les musées de sciences: quels statuts pour le visiteur scolaire?, Recherches en Didactiques, $\mathrm{n}^{\circ} 11$, p. 97-110.

COHEN-AZRIA C. (2012), « La visite scolaire au musée comme objet de construction du chercheur », Recherches, $\mathrm{n}^{\circ}$ 57, p. 159-170.

COHEN-AzRia C., Dias-ChiarutTini A. (2013), "Contenus et disciplines et aux frontières des institutions », Communication au séminaire de l'équipe Théodile, le 17 mai 2013.

COHEN-AZRIA C., DiAs-ChIARUTTINi A. (2015), « Analyser les contenus en jeu dans la visite scolaire au Musée : questions méthodologiques », dans Daunay B., Fluckiger C., Hassan, R., Les Contenus d'enseignement et d'apprentissage. Les apports de Théodile, Presses Universitaires de Bordeaux, p. 109-119.

DiAS-CHIARUTTINI A. (2012), «L'enseignement du Français aux frontières de l'extrascolaire », Recherches, $\mathrm{n}^{\circ}$ 57, p. 23-39.

DiAS-CHIARUTTINI A. (2015), « Lecture d'une l'œuvre d'art au musée d'art et en classe de Français : approche didactique $»$, Spirale, $\mathrm{n}^{\circ} 56$, p. 19-32.

GuICHARD J., (1989), «Démarche pédagogique et autonomie de l'enfant dans l'exposition scientifique », Aster n' 9, p. 17-42.

GUiChard J., MARTINAND J.-L., (2000), Médiatique des sciences, Paris, PUF.

JACOBI D., COPPEY O. (1995), «Introduction - Musée et éducation : au-delà du consensus, la recherche du partenariat », Publics et Musées, ${ }^{\circ}$ 7, p. 10-22.

LEFEBVRE B., LeFEBVRE H., (1991), «Le visiteur, le guide et l'éducation », Revue canadienne de l'éducation, $\mathrm{n}^{\circ} 16-3$, p. 331-337.

Triquet E., ClÉMent P., (1990), Confrontation d'imaginaires lors de la genèse d'une exposition, Actes JIES n ${ }^{\circ} 12$, p. $93-100$

Veron E., LeVAsseur M. (1983), Ethnographie de l'exposition : l'espace, le corps, le sens, Paris, B.P.I./Centre Pompidou. 


\section{Annexe}

1) Que penses-tu avoir appris pendant cette visite?

2) Pendant cette sortie scolaire quelle(s) matière(s) penses-tu avoir travaillées ? Qu'est-ce qui te fait penser ça?

3) Est-ce que ton professeur a précisé pourquoi il organisait cette visite ? OUI / NON - si oui, marque ici ce qu'il a dit, d'après ton souvenir : travailles?

4) Pendant cette sortie scolaire, quelle(s) matière(s) penses-tu que ton professeur voulait que tu

5) Es-tu déjà venu dans ce musée ? OUI / NON

Avec qui ? famille / école / centre de loisirs / autres

6) As-tu déjà visité d'autres musées ? OUI / NON Si oui, lesquels :

Avec qui ? famille / école / centre de loisirs / autres :

7) À ton avis, est-ce la même chose de visiter un musée en famille et avec l'école ? OUI / NON Pourquoi?

8) Donne-nous ton avis sur les visites (en cochant la case quand tu es d'accord) :

Avec la famille :

\begin{tabular}{|l|l|l|l|l|}
\hline & toujours & souvent & parfois & jamais \\
\hline J'ai du plaisir & & & & \\
\hline Je comprends & & & & \\
\hline Je m'ennuie & & & & \\
\hline Je suis fatigué & & & & \\
\hline Ça m'intéresse & & & & \\
\hline J'apprends & & & & \\
\hline On m'explique & & & \\
\hline Autres : ..................... & & & & \\
\hline
\end{tabular}

Idem avec l'école, le centre de loisirs,

9) Est-ce que tu préfères les visites avec ou sans guide ? (Entoure la réponse qui te convient) :

\begin{tabular}{|c|c|c|c|c|c|c|}
\hline & \multicolumn{2}{|c|}{ AVEC L'ÉCOLE } & \multicolumn{2}{|c|}{ AVEC LA FAMILLE } & \multicolumn{2}{|c|}{$\begin{array}{l}\text { AVEC LE CENTRE DE } \\
\text { LOISIRS }\end{array}$} \\
\hline & Avec guide & Sans guide & Avec guide & Sans guide & Avec guide & Sans guide \\
\hline $\begin{array}{l}\text { Explique } \\
\text { pourquoi }\end{array}$ & & & & & & \\
\hline
\end{tabular}

10) Est-ce que les visites dans les musées c'est important pour apprendre des choses en... (Coche la case quand tu es d'accord) :

\begin{tabular}{|l|l|l|l|}
\hline & Très important & Important & Pas important \\
\hline Mathématiques & & & \\
\hline Français & & & \\
\hline Sciences & & & \\
\hline Histoire & & & \\
\hline Géographie & & & \\
\hline Sport & & & \\
\hline Anglais (ou autres langues) & & & \\
\hline Technologie & & & \\
\hline Arts & & & \\
\hline
\end{tabular}

Explique pourquoi?

11) Est-ce que tu préfères les visites préparées avant en classe ? OUI / NON - Pourquoi ?

12) Est-ce que tu aimes qu'on reparle des visites après en classe ? OUI / NON - Pourquoi ?

13) Au musée et à l'école est-ce que tu apprends... (Coche la case quand tu es d'accord) :

\begin{tabular}{|l|l|l|l|}
\hline & OUI & NON & Pourquoi ? \\
\hline ... les mêmes choses ? & & & \\
\hline ...de la même façon? & & & \\
\hline
\end{tabular}


14) Est-ce que tu penses que c'est important de lire ce qu'il y a dans le musée quand ...

\begin{tabular}{|l|l|l|l|}
\hline & OUI & NON & Pourquoi ? \\
\hline$\ldots$ tu fais une visite scolaire ? & & & \\
\hline$\ldots$ tu fais une visite avec ta famille? & & & \\
\hline$\ldots$ tu fais une visite avec un centre de loisir? & & & \\
\hline
\end{tabular}

15) Est-ce que tu penses que c'est important d'écrire quand tu fais une visite au musée avec...

\begin{tabular}{|l|l|l|l|}
\hline & OUI & NON & Pourquoi ? \\
\hline$\ldots$ l'école ? & & & \\
\hline$\ldots$ ta famille ? & & & \\
\hline ...un centre de loisir? & & & \\
\hline
\end{tabular}

16) Raconte ton meilleur ou ton pire souvenir de visite au musée :

17) Pour toi, comment se passerait une visite idéale d'un musée ?

18) À quoi te servent les visites scolaires dans les musées?

Entoure les trois réponses les plus importantes pour toi

A rien / A travailler / A comprendre /A te comporter différemment par rapport à ce que tu dois faire dans la classe / A apprendre des choses différentes que dans la classe/ A apprendre autrement qu'à l'école / A apprendre/ A jouer avec tes camarades/ A voir ton professeur autrement / A t'amuser / A voir des objets A voir tes camarades autrement/ A découvrir / A te poser des questions

MERCI BEAUCOUP - Si tu as envie d'ajouter quelque chose, n'hésite pas : 